\author{
Потенциал кардиоскрининга на предприятии нефтегазового сектора \\ Симонян М.А. ${ }^{1}$, Герасимов С.Н. ${ }^{2}$, Буланов В.Е. ${ }^{2}$, Нестеров И.А. ${ }^{3}$, Посненкова О.М. ${ }^{1}$ \\ ${ }^{1}$ ФГБОУ ВО Саратовский ГМУ им. В.И. Разумовского Минздрава России, Саратов, Россия \\ 2 ООО «Медис», Саратов, Россия \\ ${ }^{3}$ Филиал 21477 ВМКГ МО РФ, Петропавловск-Камчатский, Россия
}

\begin{abstract}
Резюме
Цель: сравнить результаты обследования работников предприятия нефтегазового сектора, полученные в ходе ежегодного периодического медицинского осмотра, с результатами кардиоскрининга.

Материал и методы - В 2018 году кардиологом обследовано 47 сотрудников крупного предприятия нефтегазового сектора (64\% мужчины, средний возраст $52 \pm 8$ года). За 6 месяцев до кардиоскрининга все работники прошли ежегодный периодический медицинский осмотр. Анализировались данные опроса, лабораторного и инструментального исследования (глюкоза, липидограмма, данные ЭКГ). Сравнивались установленные в ходе периодического медицинского осмотра и осмотра кардиолога факторы риска, уровень риска по шкале SCORE, диагнозы, рекомендации.

Результаты - В изучаемой группе гиперхолестеринемия выявлена у 39 работников (83\%). Гипергликемия на момент обследования была выявлена у 30 человек (64\%). Признаки гипертрофии левого желудочка на ЭКГ выявлены у 6 пациентов (13\%). Диагноз АГ установлен кардиологом у 35 человек (75\%) против 26 человек (55\%), которые были выявлены терапевтом, сахарный диабет (СД) - у 8 человек (17\%) против 4 человек (8,5\%), курение - у 20 обследованных (43\%) против 16 (34\%) (р < 0,001). Средние значения систолического АД по результатам кардиоскрининга составили $152 \pm 20$ мм рт.ст. против $133 \pm 24$ мм рт.ст. (р < 0,001). К категории высокого и очень высокого риска фатальных осложнений по шкале SCORE по результатам осмотра кардиолога отнесены 26 человек (55\%) против 15 человек (32\%) $(p<0,001)$.

Заключение - На предприятиях с вредными условиями труда необходимо более тщательное выделение групп высокого сердечнососудистого риска в рамках кардиоскрининга.

Ключевые слова: факторы риска развития сердечно-сосудистых заболеваний, предприятие нефтегазового сектора, периодический медицинский осмотр, кардиоскрининг.

Библиографическая ссылка: Симонян М.А., Герасимов С.Н., Буланов В.Е., Нестеров И.А., Посненкова О.М. Потенциал кардиоскрининга на предприятии нефтегазового сектора. Кардио-ИТ 2020; 7(1): е0101.

Поступила в редакцию 20 апреля 2020. Принята в печать 26 мая 2020.

(C) 2020, Симонян М.А., Герасимов С.Н., Буланов В.Е., Нестеров И.А., Посненкова О.М.

Ответственный автор: Посненкова Олга Михайловна. Адрес для переписки: ФГБОУ ВО Саратовский ГМУ им. В.И. Разумовского Минздрава России, ул. Большая Казачья 112, Саратов, 410012, , Российская Федерация. Тел.: +7 (905) 380-39-07. E-mail: posnenkova@cardio-it.ru
\end{abstract}

Original article

\title{
The potential of cardioscreening in oil and gas refinery
}

\author{
Simonyan M.A. ${ }^{1}$, Gerasimov S.N. ${ }^{2}$, Bulanov V.E. ${ }^{2}$, Nesterov I.A. ${ }^{3}$, Posnenkova O.M. ${ }^{1}$ \\ ${ }^{1}$ Saratov State Medical University, Saratov, Russia \\ ${ }^{2}$ LLC "Medis", Saratov, Russia \\ ${ }^{3}$ Branch Office \#2, 1477 Naval Clinical Hospital, Petropavlovsk-Kamchatsky, Russia
}

\begin{abstract}
Aim: to compare the results of examination conducted among oil and gas refinery employees, obtained during yearly periodic medical examination with the results obtained during cardioscreening.

Material and Methods - 47 employees (64\% - men, mean age $52 \pm 8$ yrs) of large oil and gas refinery were examined by cardiologist in 2018. Six months before the cardioscreening all employees underwent yearly periodic medical examination. The data of anamnesis, laboratory and instrumental findings (blood glucose, lipids, ECG) of examinees were analyzed. Revealed risk factors, the levels of risk SCORE, diagnosis and recommendations made during periodic medical examination and during cardioscreening were compared.

Results - In the studied group dyslipidemia was observed in 39 employees (83\%). Elevated blood glucose was revealed in 30 examinees (64\%). Left ventricle hypertrophy on ECG was determined in 6 patients (13\%). Hypertension was diagnosed in 35 subjects (75\%) by cardiologist vs 26 subjects (55\%) by therapeutist, diabetes mellitus was diagnosed in $8(15 \%)$ vs $4(8,5 \%)$ subjects by cardiologist and therapeutist correspondingly, smoking - in $20(43 \%)$ and $16(34 \%)$ of employees correspondingly $(p<0,001)$. Mean systolic BP was $152 \pm 20$ $\mathrm{mm} \mathrm{Hg}$ during cardioscreening versus $133 \pm 24 \mathrm{~mm} \mathrm{Hg}$ during periodic medical examination $(p<0,001)$. High and very high risk SCORE have had 26 subjects (55\%) according the data of cardiologist and 15 subjects $(32 \%)$ according the data of therapeutist $(p<0,001)$.

Conclusion - In the companies with abusive working conditions it is necessary to distinguish high and very high cardiovascular risk groups by means of cardioscreening.
\end{abstract}

Keywords: cardiovascular risk factors, oil and gas refinery, periodic medical examination, cardioscreening. 

7(1): e0101.

Received 20 April 2020. Accepted 26 May 2020.

(C) 2020, Simonyan M.A., Gerasimov S.N., Bulanov V.E., Nesterov I.A., Posnenkova O.M.

Corresponding author: Olga M. Posnenkova. Address: Saratov State Medical University named after V. I. Razumovsky, Bolshaya Kazachia st., 112, Saratov, 410012, Russia. Phone: +7 (905) 380-39-07. E-mail: posnenkova@cardio-it.ru

\section{Введение}

Реализация программы всеобщей диспансеризации на территории Российской Федерации позволила упорядочить на уровне первичного звена мероприятия профилактики наиболее значимых хронических неинфекционных заболеваний (ХНИЗ) среди неорганизованных групп взрослого населения [1-4]. В последнее время фокус системных воздействий, направленных на предотвращение ХНИЗ, стал смещаться на работников предприятий $[5,6]$. Это объясняется сравнительной простотой наблюдения за организованным контингентом и экономической эффективностью таких превентивных мер [7-9]. Особого внимания требуют сотрудники предприятий, на которых имеются те или иные вредные условия труда. Согласно Приказу Министерства здравоохранения и социального развития Российской Федерации от 12 апреля 2011 г. №302н «Об утверждении перечней вредных и (или) опасных производственных факторов и работ, при выполнении которых проводятся предварительные и периодические медицинские осмотры (обследования), и порядка проведения обязательных предварительных и периодических медицинских осмотров (обследований) работников, занятых на тяжелых работах и на работах с вредными и (или) опасными условиями труда» сотрудники таких предприятий обязаны проходить периодические медицинские осмотры. Традиционно целью таких осмотров было своевременное выявление и предотвращение развития профессиональных заболеваний, оценка соответствия условий труда работника состоянию его здоровья. Однако, как на предприятиях с вредными условиями, так и в неорганизованной популяции, ведущей причиной смертности остаются сердечно - сосудистые заболевания, к которым приводят традиционные факторы риска, являющиеся следствием нездорового образа жизни. Организация медицинской помощи на предприятии облегчает задачи профилактики. Обязательные ежегодные периодические осмотры позволяют своевременно выявить на ранней стадии ХНИЗ, а также наиболее значимые факторы риска их развития. С 2019 года в рамках периодических медицинских осмотров на предприятиях проводится анкетирование по опроснику, разработанному для диспансеризации. Вопросы анкеты направлены на выявление симптомов ХНИЗ и основных факторов риска их развития. Периодический медицинский осмотр осуществляет терапевт с участием врачей-специалистов утвержденных приказом №302н, в число которых, как ни странно, не входит кардиолог. Однако некоторые предприятия в рамках программы дополнительного медицинского страхования готовы оплачивать для работников консультацию специалистакардиолога.

\section{Цель}

Сравнить результаты обследования работников предприятия нефтегазового сектора, полученные в ходе ежегодного периодического медицинского осмотра, с результатами кардиоскрининга.

\section{Материал и методы}

В 2018 году осмотрено кардиологом 47 человек (30 мужчин и 17 женщин) в возрасте от 28 до 64 лет (средний возраст $52 \pm 8$ года). Все обследованные являются сотрудниками одного из крупных предприятий нефтегазового сектора, расположенного в г. Саратове. Работа их не связана непосредственно с вредным производством. На момент осмотра кардиологом все работники уже прошли ежегодный периодический медицинский осмотр. Кардиоскрининг проводился однократно среди работников, у кого диагностированы сердечно - сосудистые заболевания (ССЗ) или выявлены факторы риска их развития. Отбор пациентов для кардиоскрининга проводился терапевтом. Периодический медицинский осмотр и кардиоскрининг проводился одной и той же организацией дополнительного медицинского страхования.

На каждого пациента по результатам периодического осмотра заполнялся лист учета результатов скринингобследования, разработанный в организации дополнительного медицинского страхования в рамках алгоритма проведения скрининг - обследования с целью выявления факторов риска развития ССЗ. В листе учета результатов скрининг-обследования отмечались следующие данные: пол, возраст, сведения о курении, употреблении алкоголя, привычках питания, физической активности, особенностях условий труда (физические перегрузки, недостаток физической активности, вахтовая работа, частая смена часовых поясов, психологическое перенапряжение, другие стрессовые ситуации), сведения о наличии ССЗ, сахарного диабета (СД) и заболеваний других классов, данные - наличии ССЗ у близких родственников, сведения о принимаемых препаратах и регулярности терапии. На эти вопросы пациент отвечал самостоятельно или с помощью медицинской сестры в виде интервью. Также в листе учета отмечались антропометрические данные (рост, вес, окружность талии (ОТ), индекс массы тела (ИМТ)), биохимические показатели крови (общий холестерин, глюкоза, триглицериды, липопротеиды низкой (лпнП) и высокой плотности (ЛПВП)), данные объективного осмотра (частота сердечных сокращений (ЧСС), уровень артериального давления (АД)), данные ЭКГ (нормальная, аритмия, нарушения проводимости, ишемия миокарда, гипертрофия миокарда, другие изменения. Рассчитывался и фиксировался в листе учета уровень абсолютного сердечно - сосудистого риска по шкале SCORE, а также установленный врачом-терапевтом диагноз и выявленные факторы риска развития ССЗ. Лист учета результатов скрининг-обследования в совокупности с амбулаторной картой пациента (форма 025-у) анализировался врачом-кардиологом во время кардиоскрининга, который проводился спустя 6 месяцев после ежегодного 
периодического осмотра. Лабораторное и инструментальное обследование повторно не выполнялось.

Данные, полученные в ходе осмотра терапевта и кардиолога у каждого больного, были сопоставлены. Сравнивались установленные факторы риска, уровень абсолютного сердечно - сосудистого риска по шкале SCORE, диагнозы, рекомендации.

Статистическая обработка проводилась при помощи пакета программ Microsoft Office Excel 2007 и STATISTICA 7,0 (StatSoft Inc., США). Категориальные данные представлены в виде частот, выраженных в процентах. Количественные данные представлены в виде медианы и интерквартильного диапазона - Ме (25\%; 75\%). При распределении отличном от нормального, и в виде среднего со стандартным отклонением (M+-SD) для нормально распределенных данных.

Для сравнения качественных переменных (бинарных, пропорций) применялся критерий Хи-квадрат (х2). Различия групп по количественным переменным оценивались на основе критерия Манна-Уитни. Статистическая значимость отличий принималась не менее 0,05.

\section{Результаты}

Анализ листов учета результатов скрининг-обследования, заполненных в ходе ежегодного профилактического медицинского осмотра, показал, что среди всех обследованных ( $n=47) 26$ человек $(55,3 \%)$ знали о том, что они страдают артериальной гипертензией. При этом антигипертензивную терапию получали 20 человек (77\% от всех страдающих АГ). Из них 11 человек (55\%) принимали антигипертензивные препараты, 9 человек (45\%) не получали лекарственной терапии. Диагноз ИБС (перенесенный инфаркт миокарда) установлен у одного человека $(2,1 \%)$, сахарный диабет - у трех человек (6,5\%). Дискомфорт за грудиной хотя бы раз в жизни испытывали 13 человек (28\%). У близких родственников обследованных имелись следующие заболевания сердечно - сосудистой системы: АГ у 43\%, инфаркт миокарда - у 11\%, у одного больного (2\%) случай внезапной сердечной смерти в семье.

В ходе периодического медицинского осмотра установлено, что среди всех обследованных курят 16 человек (34\%), ежедневно употребляют алкоголь 6 человек (15\%). Диета значительного числа исследуемых не соответствует принципам рационального питания: 35 человек (74,5\%) указывают на преобладание в своем рационе жирной, жареной пищи, 2 человека (4,3\%) - на преобладание мучных изделий, а 28 человек (60\%) досаливают пищу. 17 человек $(36,2 \%)$ отмечают значительное психологическое напряжение на работе.

По шкале SCORE высокий и очень высокий сердечно сосудистый риск имели 15 человек (32\%).

По результатам антропометрического исследования среднее значения ИМТ в группе составило $28 \pm 4,5$ кг/м2. У 70,2\% обследованных (33 человека) ИМТ превышал 25 кг/м2, из них у 19 обследованных ИМТ был 30 кг/м2 и более, то есть имело место ожирение.

По результатам лабораторных исследований уровень общего холестерина в группе составил 6,30 (5,70; 7,40) ммоль/л, уровень ТГ - 1,25 (0,89; 2,14) ммоль/л, уровень лПВП - 1,40 (1,16; 1,84) ммоль/л, лПнП - 3,90 (3,30; 4,60) ммоль/л. Гиперхолестеринемия выявлена у 39 человек (83\%). Уровень глюкозы крови составил 5,80 $(5,30 ; 6,30)$ ммоль/л. Гипергликемия (уровень глюкозы крови $\geq 5,6$ ммоль/л) на момент обследования была выявлена у 30 человек (64\%). Признаки гипертрофии левого желудочка на ЭКГ выявлены у 6 пациентов (13\%). Прочие патологические изменения на ЭКГ в исследуемой группе не выявлены.

По результатам осмотра врачом-кардиологом этих же больных спустя 6 месяцев после ежегодного планового обследования отмечается расхождение ряда показателей опроса и объективного осмотра (таблица). Так, кардиологом установлено, что среди обследуемых АГ страдают 35 человек (75\%) против 26 человек (55\%), которые были выявлены терапевтом ( $p<0,001)$. При этом антигипертензивную терапию получают 12 человек из 35 (25,5\%) по данным кардиоскрининга против 11 человек из 26 (55\%) по данным листа учета результатов периодического медицинского осмотра. ИБС страдает 1 человек (2,12\%) по данным осмотра обоих специалистов. Диагноз сахарный диабет (СД) по данным кардиолога имеют 8 человек (17\%) против 4 человек (8,5\%) по данным периодического осмотра $(p<0,001)$. Факт курения установлен кардиологом у 20 обследованных (43\%), а терапевтом - у 16 (34\%) (p < 0,001). Средние значения систолического АД по результатам осмотра кардиолога составили $152 \pm 20$ мм рт.ст. против $133 \pm 24$ мм рт.ст. ( $<0,001)$. Обращает на себя внимание отсутствие данных об уровне диастолического АД у большинства больных по данным периодического осмотра (91\%). Среди 4 пациентов, у которых присутствовали в амбулаторной карте и листе учета сведения об уровне диастолического АД, во всех случаях он равнялся 90 мм рт. ст. По данным осмотра кардиолога уровень диастолического АД составил $99 \pm 4$ мм рт. ст.

Диагноз "Ожирение» установлен кардиологом у $30 \%$ обследованных (14 человек) против 19 человек (40\%) по результатам периодического осмотра. Еще у 11 работников $(23,4 \%)$ кардиологом было отмечено наличие метаболического синдрома. В заключении терапевта по результатам периодического медицинского осмотра, а также по данным листа учета результатов скрининг-обследования для выявления факторов риска развития ССЗ метаболический синдром не был выявлен ни у одного работника.

К категории высокого и очень высокого риска фатальных осложнений по шкале SCORE по результатам осмотра терапевта были отнесены 15 человек (32\%), а по результатам осмотра кардиолога - 26 человек (55\%).

Амбулаторно рекомендовано проведение суточного мониторирования АД (СМАД) 29 исследуемым (62\%), допплерэхокардиографии 11 (23,4\%), холтеровского мониторирования 3 (6,4\%). При прохождении периодического медицинского осмотра такие методы обследования не были рекомендованы ни одному пациенту.

Медикаментозная антигипертензивная терапия рекомендована кардиологом 22 работникам (47\%), липидснижающая терапия - 31 работнику (66\%), препараты ацетилсалициловой кислоты - 2 работникам (4,3\%). Кроме этого, кардиологом были также даны рекомендации по немедикаментозным методам снижения уровня АД, холестерина и веса: бессолевая диета, снижение потребления жиров животного происхождения, нормализация режима труда и отдыха, отказ от курения и алкоголя, что было зафиксировано в амбулаторной карте работника. 
Таблица. Сравнение результатов осмотра работников нефтегазового сектора ( $\mathrm{n=47)}$ терапевтом и кардиологом

\begin{tabular}{|l|l|l|l|}
\hline Показатель & $\begin{array}{l}\text { Осмотр } \\
\text { терапевта }\end{array}$ & $\begin{array}{l}\text { Осмотр } \\
\text { кардиолога }\end{array}$ & $\begin{array}{l}\text { Значимость } \\
\text { отличий }\end{array}$ \\
\hline Курение & $\begin{array}{l}16 \text { чел. } \\
\text { (34\%) }\end{array}$ & 20 чел. (43\%) & $\mathrm{p}<0,001$ \\
\hline $\begin{array}{l}\text { Ожирение } \\
\text { (диагноз) }\end{array}$ & $\begin{array}{l}19 \text { чел. } \\
\text { (40\%) }\end{array}$ & 14 чел. (30\%) & $\mathrm{p}<0,001$ \\
\hline Диагноз АГ & $\begin{array}{l}26 \text { чел. } \\
\text { (55\%) }\end{array}$ & 35 чел. (74,5\%) & $\mathrm{p}<0,001$ \\
\hline Диагноз ИБС & 1 чел. (2\%) & 1 чел. (2\%) & - \\
\hline Диагноз СД & 4 чел. (8,5\%) & 8 чел. (17\%) & $\mathrm{p}<0,001$ \\
\hline $\begin{array}{l}\text { Средние } \\
\text { значения САД }\end{array}$ & $\begin{array}{l}133 \pm 24 \text { мм } \\
\text { рт.ст }\end{array}$ & $\begin{array}{l}152 \pm 20 \text { мм } \\
\text { рт.ст. }\end{array}$ & $\mathrm{p}<0,001$ \\
\hline $\begin{array}{l}\text { Высокий и } \\
\text { очень } \\
\text { Высокий риск } \\
\text { ССОкЕ }\end{array}$ & $\begin{array}{l}15 \text { чел. } \\
(32 \%)\end{array}$ & 26 чел. (55\%) & $\mathrm{p}<0,001$ \\
\hline
\end{tabular}

Динамическое наблюдение по результатам кардиоскрининга было рекомендовано 27 сотрудникам $(57,5 \%)$.

Анализ медицинской документации показал, что терапевт в ходе периодического медицинского осмотра не давал рекомендаций по дополнительному обследованию, лечению и динамическому наблюдению ни одному пациенту из анализируемой группы.

\section{Обсуждение}

Результаты кардиоскрининга среди работников нефтегазового сектора в 2018 году свидетельствуют о высокой распространенности среди них факторов риска развития ССЗ. Так, у подавляющего большинства работников (83\%) выявлена дислипидемия, 3/4 страдали АГ, 2/3 имели лишний вес. Не менее чем у трети обследованных отмечалось ожирение. Нарушения углеводного обмена (сахарный диабет или метаболический синдром) зафиксированы у $40 \%$ работников. Около половины курили. По сравнению с данными многоцентрового российского исследования ЭССЕ-РФ (Эпидемиология Сердечно-Сосудистых заболеваний в различных регионах Российской Федерации) в изученной части организованного коллектива вдвое чаще, чем в общей популяции встречалось курение, на 50\% чаще - АГ [10]. По сравнению с результатами российского эпидемиологического исследования NATION [11], где распространенность нарушений углеводного обмена - СД II типа и преддиабета среди взрослого населения составила $24,7 \%$ (5,4\% - СД и 19,3\% преддиабет), в анализируемой группе частота выявленных отклонений была практически вдвое выше.

В ходе сравнения результатов кардиоскрининга с данными медицинской документации, заполняемой в ходе ежегодного периодического осмотра, отмечено существенное расхождение по частоте выявления факторов риска, диагнозам, присваиваемой категории сердечно-сосудистого риска и качеству рекомендаций. Несмотря на то, что в изучаемой организации дополнительного медицинского страхования уделяется внимание не только выявлению профессиональных заболеваний, но и традиционных факторов риска развития ССЗ, в частности, в 2018 году в инициативном порядке реализуется алгоритм проведения скринингобследования с целью выявления факторов риска развития
СС3, ряд пациентов высокого риска ускользает из-под наблюдения.

Как показали результаты осмотра кардиолога, большинство работников требуют дообследования и регулярного динамического наблюдения. Формат ежегодного профосмотра позволяет лишь констатировать естественную эволюцию от факторов риска к заболеваниям, но не бороться с ними планомерно. Результаты реализуемых корпоративных программ [12, 13] и отечественных научных исследований в области активной профилактики на рабочем месте свидетельствуют о ее клинической и экономической целесообразности $[9,14-16]$.

Учитывая, что работодатель оплачивает только ежегодный периодический медицинский осмотр, нужны новые низкозатратные технологии взаимодействия с работниками, в том числе на основе информационных технологий с применением дистанционного наблюдения без отрыва от производства.

\section{Заключение}

На предприятиях с вредными условиями труда необходимо более тщательное выделение групп высокого сердечно-сосудистого риска среди работников предприятий (кардиоскрининг) и внедрение низкозатратных способов долгосрочного динамического наблюдения, в том числе на основе информационных и телемедицинских технологий. 


\section{Литература}

1. Погосова Н.В., Юферева Ю.М., Самородская И.В., Бойцов С.А. Профилактический скрининг: все за и против. Кардиоваскулярная терапия и профилактика 2016; 15(3): 4-13 http://dx.doi.org/10.15829/1728-8800-2016-3-4-13.

2. Яковлева Т.В., Вылегжанин С.В., Бойцов С.А. и др. Диспансеризация взрослого населения Российской Федерации: первый год реализации, опыт, результаты, перспективы. Социальные аспекты здоровья населения 2014; 4(38). http://vestnik.mednet.ru/content/view/579/30/. Дата обращения: 15.04.2020.

3. Концевая А.В., Калинина А.М., Бойцов С.А., Ипатов П.В. Социальноэкономическая эффективность диспансеризации 2013 года в Российской Федерации. Кардиоваскулярная терапия и профилактика 2014; 3: 4-10.

4. Калинина А.М., Ипатов П.В., Кушунина Д.В. и др. Результаты выявления болезней системы кровообращения при диспансеризации взрослого населения: опыт первых 2 лет. Терапевтический архив 2016; 1: 46-52.

5. Копылова Г., Недобой И., Феоктистова Е. и др. Здоровье на рабочем месте, сборник корпоративных практик / РСПП, Москва, 2011 г. -92 стр.

6. Корпоративные модельные программы «Укрепление здоровья работающиху

https://static-

1.rosminzdrav.ru/system/attachments/attaches/000/046/785/original \%D0\%9A\%D0\%BE\%D1\%80\%D0\%BF\%D0\%BE\%D1\%80\%D0\%B0\%D1\%8 2\%D0\%B8\%D0\%B2\%D0\%BD\%D1\%8B\%D0\%B5 \%D0\%BC\%D0\%BE\%D0 \%B4\%D0\%B5\%D0\%BB\%D1\%8C\%D0\%BD\%D1\%8B\%D0\%B5 \%D0\%BF\% D1\%80\%D0\%BE\%D0\%B3\%D1\%80\%D0\%B0\%D0\%BC\%D0\%BC\%D1\%8B \%D0\%A3\%D0\%BA\%D1\%80\%D0\%B5\%D0\%BF\%D0\%BB\%D0\%B5\%D0\%B D\%D0\%B8\%D0\%B5 \%D0\%BE\%D0\%B1\%D1\%89\%D0\%B5\%D1\%81\%D1\% 82\%D0\%B2\%D0\%B5\%D0\%BD\%D0\%BD\%D0\%BE\%D0\%B3\%D0\%BE \%D0 \%B7\%D0\%B4\%D0\%BE\%D1\%80\%D0\%BE\%D0\%B2\%D1\%8C\%D1\%8F.pdf ?1565881637 Дата обращения 15.04.2020.

7. Naydeck BL, Pearson JA, Day BT, Goetzel RZ. The impact of the highmark employee wellness programs on 4-year healthcare costs. J Occup Environ Med 2008; 50: 146-156. https://doi.org/10.1097/JOM.0b013e3181617855.

8. Dement JM, Epling C, Joyner J, Cavanaugh K. Impacts of Workplace Health Promotion and Wellness Programs on Health Care Utilization and Costs: Results From an Academic Workplace. J Occup Environ Med. 2015; 57(11): 1159-69. https://doi.org/10.1097/JOM.0000000000000555.

9. Пырикова Н.В., Концевая А.В., Осипова И.В. Клиникоэкономический анализ реализации первичной профилактики сердечно-сосудистых заболеваний в организованном коллективе. Бюллетень медицинской науки 2018; 4(12): 60-66.

10. Баланова Ю.А., Концевая А.В., Шальнова С.А. и др. Распространенность поведенческих факторов риска сердечнососудистых заболеваний в российской популяции по результатам исследования ЭССЕ-РФ. Профилактическая медицина 2014; 5: 4251.

11. Dedov I, Shestakova M, Benedetti MM, et al. Prevalence of type 2 diabetes mellitus $(\mathrm{t} 2 \mathrm{dm}$ ) in the adult Russian population (nation study). Diabetes Res Clin Pract 2016; 115: 90-95. https://doi.org/10.1016/j.diabres.2016.02.010

12. Библиотека корпоративных программ укрепления здоровья работников

https://static-

1.rosminzdrav.ru/system/attachments/attaches/000/046/753/original \%D0\%91\%D0\%B8\%D0\%B1\%D0\%BB\%D0\%B8\%D0\%BE\%D1\%82\%D0\%B 5\%D0\%BA\%D0\%B0 \%D0\%BA\%D0\%BE\%D1\%80\%D0\%BF\%D0\%BE\%D1\% 80\%D0\%B0\%D1\%82\%D0\%B8\%D0\%B2\%D0\%BD\%D1\%8B\%D1\%85 \%D0 \%BF\%D1\%80\%D0\%BE\%D0\%B3\%D1\%80\%D0\%B0\%D0\%BC\%D0\%BC.pdf ?1565693486. Дата обращения 17.04.2020

13. Кейсы корпоративных программ укрепления здоровья работников https://medvestnik.ru/files/download/LOSegstluXaKrkAlH741mUCILm PJsksr. Дата обращения 17.04.2020.
14. Концевая А.В. Программа профилактики хронических неинфекционных заболеваний в ОАО «Сбербанк России»: синтез доказательной медицины и маркетинговых технологий. Российский кардиологический журнал 2014; 9(113): 16-21.

15. Пырикова Н.В., Осипова И.В., Концевая А.В. и др. Клиникоэкономическая эффективность организационной модели первичной профилактики сердечно-сосудистых заболеваний у работников железнодорожного транспорта. Российский кардиологический журнал 2014; 6(110): 12-18.

16. Калинина А.М., Концевая А.В., Белоносова С.В. и др. Реализация программного цикла профилактики сердечно-сосудистых заболеваний на рабочем месте: клиническая эффективность. Кардиоваскулярная терапия и профилактика 2010; 9(3): 90-97.

\section{References}

1. Pogosova NV, Yufereva YuM., Samorodskaya IV, Boytsov SA. Preventional screening: all pros and contras. Cardiovascular Therapy and Prevention 2016; 15(3): 4-13 http://dx.doi.org/10.15829/17288800-2016-3-4-13/

2. Yakovleva TV, Vylegzhanin SV, Boitsov SA, et al. Regular medical examination of adults in the Russian Federation: first year implementation: lessons learnt, results and perspectives. Social aspects of population health 2014; 4(38). http://vestnik.mednet.ru/content/view/579/30/. The date of application 15.04.2020.

3. Kontsevaya AV, Kalinina AM, Boytsov SA, Ipatov PV. Socio-economic effectiveness of health screening activity in 2013 in Russian Federation. Cardiovascular Therapy and Prevention 2014; 3: 4-10.

4. Kalinina AM, Ipatov PV, Kushunina DV, et al. Results of circulatory disease detection during prophylactic medical examination of the adult population: the first two years' experience. Therapeutic Archive 2016; 1: 46-52. https://doi.org/10.17116/terarkh201688146-52

5. Kopylova G., Nedoboj I., Feoktistova E., et al. Health at the Workplace, Collection of Social Practices/ RSPP, Moscow, 2011 - 92p.

6. Corporative model programs "Employees' health promotion" https://static-

1.rosminzdrav.ru/system/attachments/attaches/000/046/785/original \%D0\%9A\%D0\%BE\%D1\%80\%D0\%BF\%D0\%BE\%D1\%80\%D0\%B0\%D1\%8 2\%D0\%B8\%D0\%B2\%D0\%BD\%D1\%8B\%D0\%B5 \%D0\%BC\%D0\%BE\%D0 \%B4\%D0\%B5\%D0\%BB\%D1\%8C\%D0\%BD\%D1\%8B\%D0\%B5 \%D0\%BF\% D1\%80\%D0\%BE\%D0\%B3\%D1\%80\%D0\%B0\%D0\%BC\%D0\%BC\%D1\%8B \%D0\%A3\%D0\%BA\%D1\%80\%D0\%B5\%D0\%BF\%D0\%BB\%D0\%B5\%D0\%B D\%D0\%B8\%D0\%B5 \%D0\%BE\%D0\%B1\%D1\%89\%D0\%B5\%D1\%81\%D1\% 82\%D0\%B2\%D0\%B5\%D0\%BD\%D0\%BD\%D0\%BE\%D0\%B3\%D0\%BE_\%D0 \%B7\%D0\%B4\%D0\%BE\%D1\%80\%D0\%BE\%D0\%B2\%D1\%8C\%D1\%8F.pdf? 1565881637 The date of application 15.04.2020.

7. Naydeck BL, Pearson JA, Day BT, Goetzel RZ. The impact of the highmark employee wellness programs on 4-year healthcare costs. J Occup Environ Med 2008; 50: 146-156. https://doi.org/10.1097/JOM.0b013e3181617855.

8. Dement JM, Epling C, Joyner J, Cavanaugh K. Impacts of Workplace Health Promotion and Wellness Programs on Health Care Utilization and Costs: Results From an Academic Workplace. J Occup Environ Med. 2015; 57(11): 1159-69. https://doi.org/10.1097/JOM.0000000000000555.

9. Pyrikova NV, Osipova IV, Kontsevaya AV. Clinical and economic analysis of primary prevention of cardiovascular diseases in organized body. Bulletin of Medical Science 2018; 4(12): 60-66.

10. Balanova YuA, Kontsevaya AV, Shalnova SA, et al. The prevalence of behavioral risk factors for cardiovascular diseases in the Russian population according to the results of the ESSE-RF study. Russian Journal of Preventive Medicine and Public Health 2014; 5: 42-51.

11. Dedov I, Shestakova M, Benedetti MM, et al. Prevalence of type 2 diabetes mellitus $(\mathrm{t} 2 \mathrm{dm})$ in the adult Russian population (nation study). Diabetes Res Clin Pract 2016; 115: 90-95. https://doi.org/10.1016/i.diabres.2016.02.010 
12. Bibliography of corporative programs of health promotion for employees. https://static1.rosminzdrav.ru/system/attachments/attaches/000/046/753/original \%OD0\%91\%D0\%B8\%D0\%B1\%D0\%BB\%D0\%B8\%D0\%BE\%D1\%82\%D0\%B 5\%D0\%BA\%D0\%B0 \%D0\%BA\%D0\%BE\%D1\%80\%D0\%BF\%D0\%BE\%D1\% 80\%D0\%B0\%D1\%82\%D0\%B8\%D0\%B2\%D0\%BD\%D1\%8B\%D1\%85 \%D0 \%BF\%D1\%80\%D0\%BE\%D0\%B3\%D1\%80\%D0\%B0\%D0\%BC\%D0\%BC.pdf ?1565693486. The date of application 17.04.2020.

13. Cases of corporative programs of health promotion for employees. https://medvestnik.ru/files/download/LOSegstluXaKrkAlH741mUCILm PJsksr. The date of application 17.04.2020.

14. Kontsevaya AV. The program for prevention of chronic non-infectious diseases in JSC "Sberbank of Russia": synthesis of evidence-based medicine and marketing technologies. Russ J Cardiol 2014; 9(113): 1621.

15. Pyrikova NV, Osipova IV, Kontsevaya AV, et al. Clinical and economic efficacy of primary prediction organizational model for cardiovascular diseases in railway system workers. Russ I Cardiol 2014; 6(110): 12-18.

16. Kalinina AM, Kontsevaya AV, Belonosova SV, et al. Workplace program of cardiovascular prevention: clinical effectiveness. Cardiovascular Therapy and Prevention 2010; 9(3): 90-97.

\section{Информация об авторах:}

Симонян Маргарита Андреевна - младший научный сотрудник отдела атерослероза и хронической ишемической болезни сердца, НИИ кардиологии, ФГБОУ ВО Саратовский ГМУ им. В.И. Разумовского Минздрава России, Саратов, Россия. http://orcid.org/0000-0002-98663069.

Герасимов Станислав Николаевич - директор филиала ООО «Медис» в г. Саратов, Саратов, Россия.

Буланов Виктор Евгеньевич - канд. мед. наук, заместитель генерального директора ООО «Медис» по медицинской деятельности, Москва, Россия.

Нестеров Иван Александрович - начальник отделения, филиал №2 ФГКУ «1477 ВМКГ» Минобороны России, Петропавловск-Камчатский, Россия.

Посненкова Ольга Михайловна - д-р мед. наук, ведущий научный сотрудник, отдел атеросклероза и хронической ишемической болезни сердца, НИИ кардиологии, ФГБОУ ВО Саратовский ГМУ им. В.И Разумовского Минздрава России, Саратов, Россия. http://orcid.org/0000-0001-5311-005X.

Authors:

Margarita A. Simonyan - Junior Researcher of Department of Atherosclerosis and Chronic Coronary Artery Disease, Institute of Cardiological Research, Saratov State Medical University, Saratov, Russia. http://orcid.org/0000-0002-9866-3069.

Stanislav N. Gerasimov - Head of Saratov Branch Office, LLC "Medis", Saratov, Russia.

Viktor E. Bulanov - MD, PhD, Vice-Chief on Medicine, LLC "Medis", Moscow, Russia.

Ivan A. Nesterov - Head of Department, Branch Office \#2, 1477 Naval Clinical Hospital, Petropavlovsk-Kamchatsky, Russia.

Olga M. Posnenkova - MD, DSc, Leading Researcher, Department of New Cardiological Informational Technologies, Research Institute of Cardiology, Saratov State Medical University, Saratov, Russia. http://orcid.org/00000001-5311-005X. 